\title{
Otitis, Sinusitis, and Mastoiditis
}

\section{Ear or Facial Pain Following a Common Cold}

Winter S. Berry

4.1 Otitis - 39

4.1.1 Introduction - 39

4.2 Definitions - 39

4.3 Basic Concepts - 39

4.3.1 Otitis Externa - 39

4.3.2 Acute Otitis Media - 40

4.3.3 Risk Factors -40

4.3.4 Microbiologic Causes of AOM - 40

4.3.5 Approach to the Diagnosis of AOM - 41

4.3.6 Differential Diagnosis of AOM - 41

4.3.7 Treatment of AOM - 41

4.3.8 Complications of AOM - 43

4.3.9 Follow-Up - 43

4.4 Sinusitis -44

4.4.1 Introduction - 44

4.5 Definitions - 44

4.5.1 Sinusitis: Inflammation of the Paranasal Sinus Cavity Mucosa -44

4.6 Basic Concepts - 45

4.6.1 Anatomy and Pathophysiology -45

4.6.2 Risk Factors -45

4.6.3 Microbiologic Causes of Acute Bacterial Rhinosinusitis - 45

4.6.4 Approach to the Diagnosis - 45

4.6.5 Differential Diagnosis of Bacterial Rhinosinusitis - 47

4.6.6 Treatment of Bacterial Rhinosinusitis -47

4.6.7 Complications of Bacterial Sinusitis -47

4.7 Mastoiditis -48

4.7.1 Introduction -48 
4.8 Definitions -48

4.9 Basic Concepts -48

4.9.1 Pathophysiology -48

4.9.2 Risk Factors for the Development of Mastoiditis - 48

4.9.3 Microbiologic Causes of Mastoiditis - 48

4.9.4 Approach to the Diagnosis of Mastoiditis - 49

4.9.5 Differential Diagnosis of Mastoiditis - 49

4.9.6 Treatment of Mastoiditis - 49

4.9.7 Complications of Mastoiditis - 50

4.10 Exercises -50

4.11 Summary -51

References - 51 


\section{Learning Objectives}

- Know the common clinical presentations for otitis, sinusitis, and mastoiditis.

- Identify common and uncommon microbiologic causes of otitis, sinusitis, and mastoiditis.

- Understand the distinguishing characteristics for acute, recurrent, and chronic clinical courses of each disease.

- List the important risk factors for developing severe infections of the paranasal sinuses.

- Outline the approach to diagnosis, including signs and symptoms that warrant laboratory or imaging evaluations.

- Describe the indications for medical and surgical treatment of otitis, sinusitis, and mastoiditis.

\subsection{Otitis}

\subsubsection{Introduction}

The term "otitis" encompasses pathology of both the middle and outer ear. It is generally divided into two categories otitis media and otitis externa. Otitis media can present either as an acute infectious process of the middle ear (acute otitis media) or as a serous noninfectious process (otitis media with effusion). Otitis externa is an infectious inflammatory condition of the external auditory canal (EAC). Approaches to the diagnosis and treatment of acute otitis media have evolved over the last several decades as new immunizations, and more antibiotic choices have become available.

\subsection{Definitions}

Otitis media with effusion (OME) - A collection of serous fluid in the middle ear space without signs of acute inflammation. OME is not an infectious process.

Chronic otitis media with effusion (COME) - A collection of serous fluid in the middle ear space that persists for more than 3 months

Acute otitis media (AOM) - An acute infection of the middle ear with signs and symptoms of acute inflammation. An effusion is also present. Recurrent AOM - Three or more episodes of AOM in a 6-month period or 4 or more episodes of AOM in a 12-month period

Otorrhea - The presence of a discharge from the ear

Chronic suppurative otitis media (CSOM) - The presence of a purulent middle ear effusion associated with otorrhea, secondary to chronic tympanic membrane perforation, for more than 6 weeks in the setting of antibiotic treatment

Otitis externa (OE) - An infection of the external auditory canal

\subsection{Basic Concepts}

\subsubsection{Otitis Externa}

Acute otitis externa develops following disruption of the epithelial cell layer of the EAC. Epithelial breakdown can be caused by excessive moisture that leads to maceration, trauma during insertion of a foreign body, occlusion by a device such as a hearing aid or earplug, or dermatologic conditions involving the EAC. Excessive moisture as a cause for breakdown or maceration of the skin that lines the EAC is very common. The frequency of otitis externa among swimmers underscores the importance of keeping the EAC dry and explains why otitis externa is commonly known as "swimmer's ear."

The presenting symptoms of otitis externa include otalgia, decreased hearing, sensation of fullness in the ear canal, pruritus, tenderness on palpation, and movement of the EAC or the pinna. Otorrhea, adjacent cervical lymph node enlargement, and local cellulitis may develop later in the course of more severe cases. The most common bacterial causes of $\mathrm{OE}$ include Pseudomonas aeruginosa and Staphylococcus aureus [1]. Typical physical examination findings include erythema and edema of the EAC with debris, cerumen, and purulent material filling the canal. Visualization of the tympanic membrane (TM) is often obstructed by the otorrhea and the swelling associated with the inflammation. An unimpaired view of a normal TM, with visible landmarks, is shown in • Fig. 4.1. The diagnosis of $\mathrm{OE}$ is made based on the history and the clinical findings. Typical otoscopic findings of OE are shown in - Fig. 4.2. When the EAC is cultured, one must be cognizant that culture results may reflect EAC flora rather than a causative organism. First-line antibiotic treatments include topical otic drops of a fluoroquinolone, such as ciprofloxacin with or without topical glucocorticoid drops. If edema of the EAC is severe, placement of a wick inside the EAC may be necessary to ensure delivery of medication to the more proximal areas of infection. Risk of recurrence of $\mathrm{OE}$ is increased in individuals with atopic dermatitis, seborrhea, immune compromise, and repeated local trauma to the area when

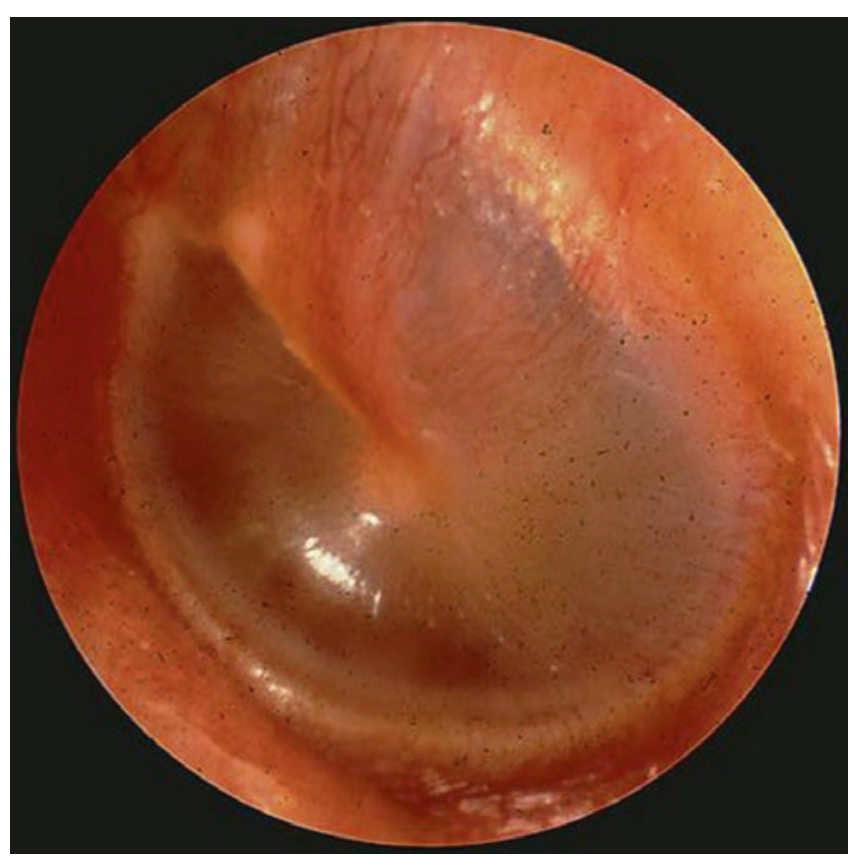

- Fig. 4.1 Normal tympanic membrane. (Courtesy of Dr. Charles Woods, SUNY Upstate Medical University) 


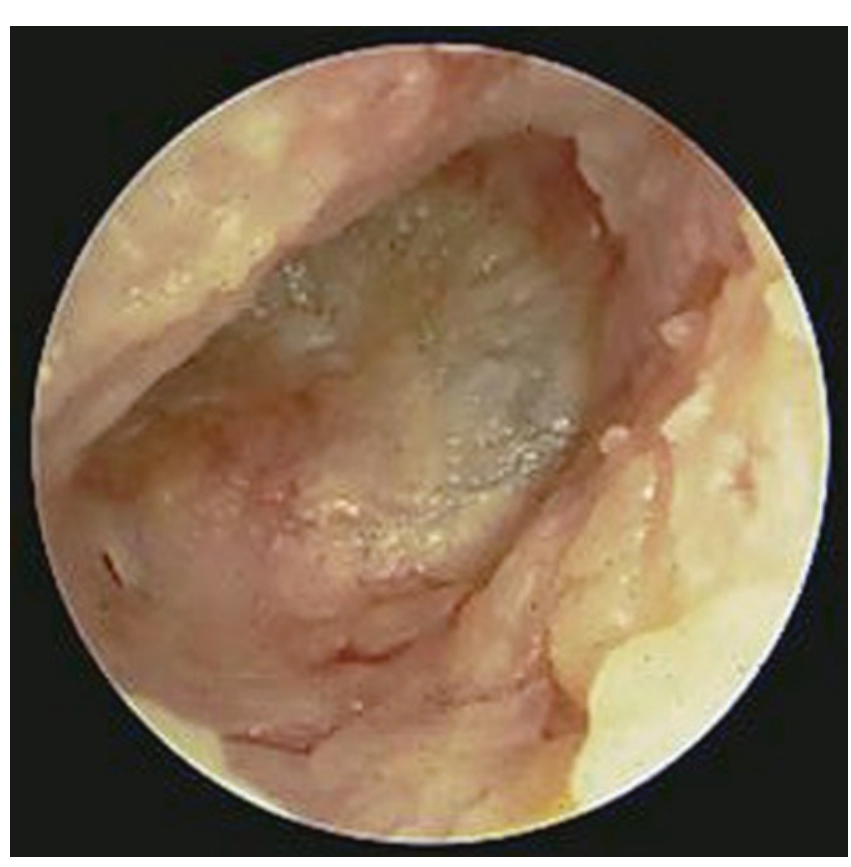

- Fig. 4.2 Otitis externa. (Courtesy of Dr. Charles Woods, SUNY Upstate Medical University)

cleaning the ear. A fungal etiology of the infection should be considered when otorrhea is prolonged, especially if topical antibacterials and a wick have been used already. Individuals who use hearing aids and those who have had recent bacterial infections are also at risk for fungal disease. The most common fungal etiologies include Aspergillus and Candida species [1].

\subsubsection{Acute Otitis Media}

The development of AOM begins when there is dysfunction of the eustachian tube. Under normal conditions, the eustachian tube allows the middle ear to drain to the pharynx and to equalize pressure between the middle ear and the environment. Impaired drainage may be present for several reasons. The anatomic position of the shorter eustachian tube in young children maintains a relatively horizontal orientation allowing the drainage to defy gravity. Adenoidal hypertrophy and anatomic anomalies of the palate can also block or impair normal drainage. The presence of gastroesophageal reflux disease, allergic rhinitis, and viral upper respiratory infections can all lead to inflammation of the eustachian tube and surrounding tissues resulting in the presence of increased secretions in the middle ear that accumulates because the eustachian tube is not fully patent [2-4]. In each of these clinical scenarios, negative pressure develops in the eustachian tube and middle ear space. The small number of bacteria normally present in those secretions replicates in the now closed space resulting in acute infection (AOM). Retained nasopharyngeal secretions are very common in individuals with otherwise uncomplicated viral respiratory infections. A subgroup of these individuals experience secondary bacterial infection because the initial viral infection and its associated inflammation allow for the closed space conditions where the bacteria can thrive.

\subsubsection{Risk Factors}

The risk factors for $\mathrm{AOM}$ include any condition that promotes eustachian tube dysfunction. The relative horizontal position of the eustachian tube during early childhood explains why the peak incidence of AOM is between 6 and 18 months of age. Additional risk factors include children who develop their first episode of AOM prior to 6 months of age, children in daycare, and the presence of atopy, adenoidal hypertrophy, chronic sinusitis, ciliary dysfunction, immunecompromising conditions, and craniofacial anomalies. Individuals with trisomy 21 are especially prone to otitis media because their eustachian tubes are short and their pharyngeal muscle tone is weak $[2,3]$.

\subsubsection{Microbiologic Causes of AOM}

An accurate description of the infectious etiologies of AOM requires that middle ear fluid is removed during acute infection and submitted for microbiologic testing. The procedure to remove the fluid, tympanocentesis, is no longer performed on a routine basis. Most of the literature that describes the microbiologic causes of AOM was published before the routine introduction of Haemophilus influenzae type B (late 1980s) and heptavalent (2000) and 13-valent (2010) conjugate pneumococcal vaccines. Rates of viral, bacterial, and mixed culture results from middle ear effusion vary significantly across those studies with rates of bacterial infection ranging between $50 \%$ and $90 \%$ of all AOM. The most common viral causes include respiratory syncytial virus; parainfluenza viruses, types 1, 2, and 3; influenza A and B viruses; adenovirus; coronaviruses; parechoviruses; and human metapneumovirus.

The most common bacterial causes of AOM include Streptococcus pneumoniae, non-typeable Haemophilus influenzae, and Moraxella catarrhalis. S. pneumoniae was unequivocally the most common bacterial agent of AOM before conjugate pneumococcal vaccine was added to the universal pediatric immunization schedule in 2000. Following vaccine introduction, non-typeable $H$. influenzae became more predominant, and "replacement" pneumococcal serotypes that were not included in the 7 - or 13-valent vaccines emerged [2-4].

Non-typeable $H$. influenzae should be suspected in the clinical setting of AOM when purulent conjunctivitis is also present. Suspicion that $H$. influenzae could be the underlying cause of the patient's condition is important, because unlike S. pneumonia, $H$. influenzae may produce a beta-lactamase, an enzyme that inactivates some of the most common antibiotics used empirically to treat AOM, such as amoxicillin. Less common bacterial etiologies of AOM include 
Streptococcus pyogenes (also known as group A streptococcus, the cause of "strep throat") and Staphylococcus aureus. S. pyogenes is more commonly seen among those older than 5 years of age. Its presentation is typically quite aggressive leading to perforation of the TM and/or accompanying mastoiditis [2]. S. aureus should be included on the list of possible infecting agents in patients who have tympanostomy tubes, as these medical devices serve as a conduit between the bacteria normally present in the EAC and the middle ear. Streptococcus agalactiae (also known as group B streptococcus) should be considered as a possible causative organism in AOM when the process is identified in neonates and very young infants.

The microbiologic culprits responsible for chronic suppurative otitis media include $S$. aureus, Pseudomonas aeruginosa, and S. pneumonia. Chronic suppurative otitis media is especially common among individuals from Southeast Asia, the Western Pacific, Africa, and Native Americans of the desert southwest [2].

\subsubsection{Approach to the Diagnosis of AOM}

AOM is a closed space infection. As the infection progresses, symptoms change from a feeling of fullness to general achiness. The pain intensifies as the pressure in the infected space increases until that pressure is relieved. Relief may come spontaneously as serous fluid is reabsorbed or when the pressure exceeds the capacity of the TM and the eardrum ruptures. Tympanocentesis can also be used as a controlled technique to remove middle ear fluid and thereby reduce the pressure. AOM causes ear pain. The pain in young children can manifest itself as fussiness, sleep disruption, or ear tugging. Fever is expected in young children with AOM, but uncommon among older children and adults with AOM. In cases where the TM ruptures, the pain is relieved, but the patient or parent will note a purulent or bloody ear discharge.

A diagnosis of AOM is often suspected while obtaining the history of the illness. The diagnosis is confirmed by finding on the physical examination. A thorough otoscopic exam, including pneumatic otoscopy and clear visualization of the TM and EAC, is essential. Presuming a diagnosis of AOM in the absence of a thorough examination leads to overdiagnosis and subsequent overuse of antibiotics. Pneumatic otoscopy should always be included to assess the mobility of the TM. Efficient pneumatic otoscopy is aided by using a speculum with a tight seal of the EAC [4]. Classic otoscopy findings of AOM include the presence of fluid in middle ear space, outward bulging of the TM secondary to the increased pressure in the middle ear space, loss of visualization of ossicle bony landmarks behind the TM, and erythema or injection of radial vessels on the TM. The middle ear effusion is purulent in AOM but must be carefully distinguished from opaque noninfectious effusions secondary to OME. The absence of erythema of the TM suggests a diagnosis of OME, not AOM. A bulging TM with decreased mobility on pneumatic otoscopy is the most specific exam finding for bacterial
AOM [4]. The presence of purulent otorrhea in the EAC is seen if a TM perforation has occurred before the time of examination. This finding must be distinguished from the edema and erythema that occur along the EAC during otitis externa. Bullae may be present on the TM in conjunction with other signs of acute inflammation. The findings are consistent with a form of AOM termed bullous myringitis. The presence of bullae does not suggest a specific microbiologic cause nor does it change the clinical approach to the condition.

In summary, the clinical diagnosis of AOM is made when there is an acute onset of symptoms, the presence of a middle ear effusion, and objective signs of acute middle ear inflammation such as a red bulging eardrum that does not move during pneumatic otoscopy [2-4].

The diagnosis of AOM does not involve any laboratory evaluation or radiographic imaging unless there is evidence for a significant complication or severe or persistent disease. Tympanocentesis with culture of the middle ear effusion is the gold standard for diagnosing the etiologic agent of AOM, but is only necessary in a small subset of cases. When necessary, referral to an otolaryngologist is considered the best practice for those who are not certified and experienced in performing the procedure. The inclination to collect samples from the EAC for microbiologic cultures, even in cases where there is frank otorrhea, is generally discouraged since the laboratory results often reflect EAC flora rather than identifying the true causative organism(s).

\subsubsection{Differential Diagnosis of AOM}

The differential diagnosis for AOM includes otalgia secondary to OME, referred pain from dental or pharyngeal disease, local herpes zoster infection, otitis externa, adjacent soft tissue infection, or eustachian tube dysfunction secondary to alternative causes.

\subsubsection{Treatment of AOM}

Recommendations for the treatment of AOM have evolved over the past several decades. Depending on the details of the clinical circumstance, current recommendations now permit a clinical observation period as an alternative to immediate antibiotic therapy for many cases. This approach is supported by the knowledge that many cases are caused by respiratory viruses and by evidence demonstrating that a reasonable proportion resolve without intervention. Ultimately, the approach used should carefully weigh patient-specific factors such as age, severity of presenting symptoms, risk factors for severe infection, history of AOM, and the patient's availability to follow up. Safety-net antibiotic prescriptions (SNAP) and wait-and-see prescription (WASP) are prescriptions provided at the time of diagnosis allowing patients and families to employ watchful waiting, with the option to fill an antibiotic prescription if the symptoms persist or worsen over the 


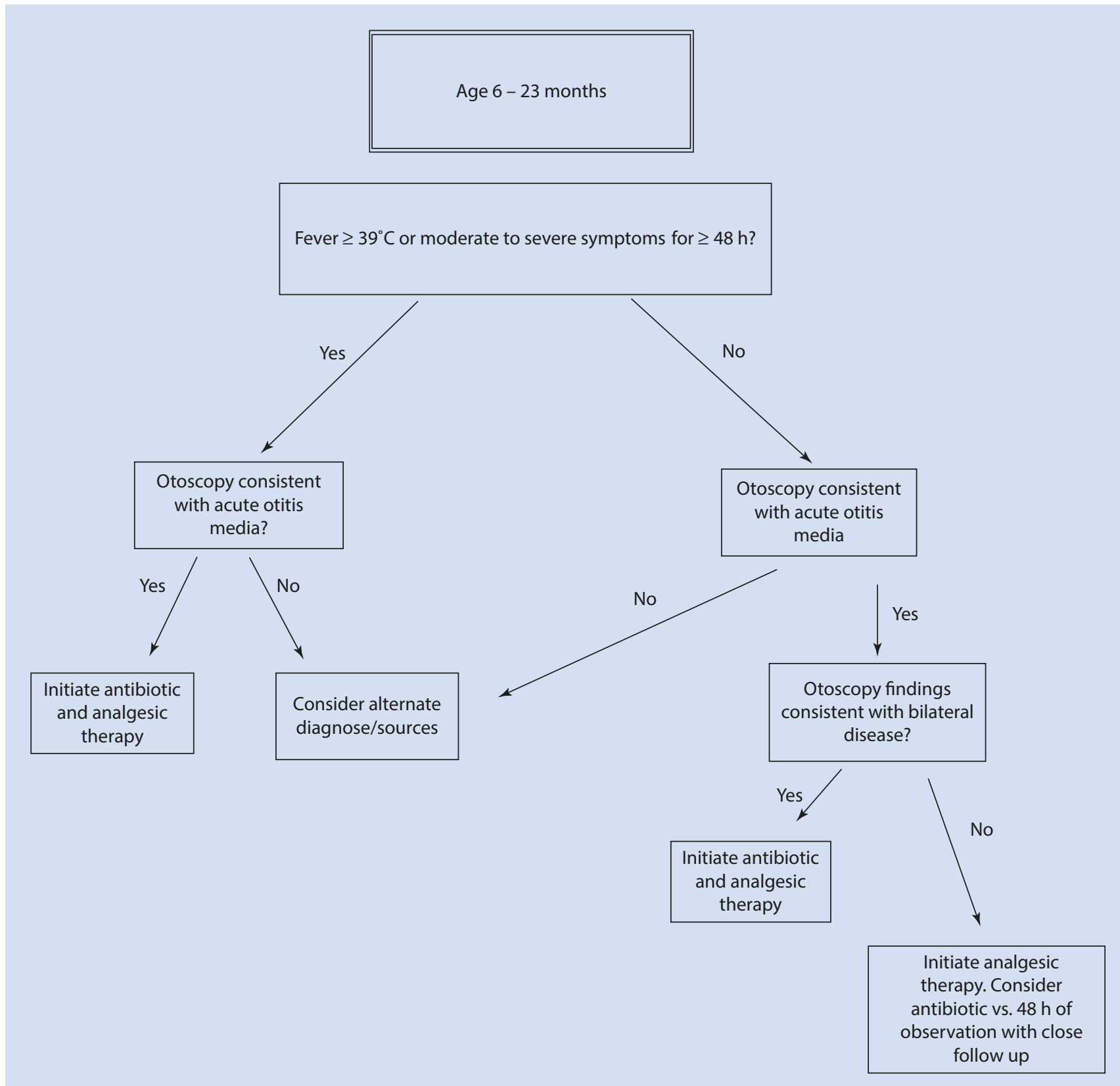

- Fig. 4.3 Treatment of AOM in patients $<24$ months of age

next 48-72 h [2-4]. Treatment approach algorithms that account for age, examination findings, and illness severity at the time of presentation are shown in - Figs. 4.3 and 4.4. Analgesic should be used regularly early in treatment course to provide symptomatic relief of otalgia $[2,4]$.

The first-line antibiotic choices for the treatment of AOM are shown in $>$ Call Out Box 4.1 [4]. High-dose amoxicillin (80$90 \mathrm{mg} / \mathrm{kg} /$ day) is recommended to overcome concerns about adequate coverage against penicillin nonsusceptible S. pneumonia. Recommendations regarding duration of antibiotic therapy are age dependent. Except for intramuscular ceftriaxone, a 10-day course is recommended for children less than 2 years of age, whereas a 7-day course can be considered for children between 2 and 5 years of age. A 5- to 7-day course is usually adequate starting at 6 years of age through adulthood [4].

Antibiotic therapy for the treatment of CSOM requires administration of topical fluoroquinolone antibiotic drops with or without glucocorticoid drops. The medications are able to reach the middle ear space by crossing through the perforation in the TM. Fluoroquinolones are used because their spectrum of activity includes the most common etiologic agents including $P$. aeruginosa. 

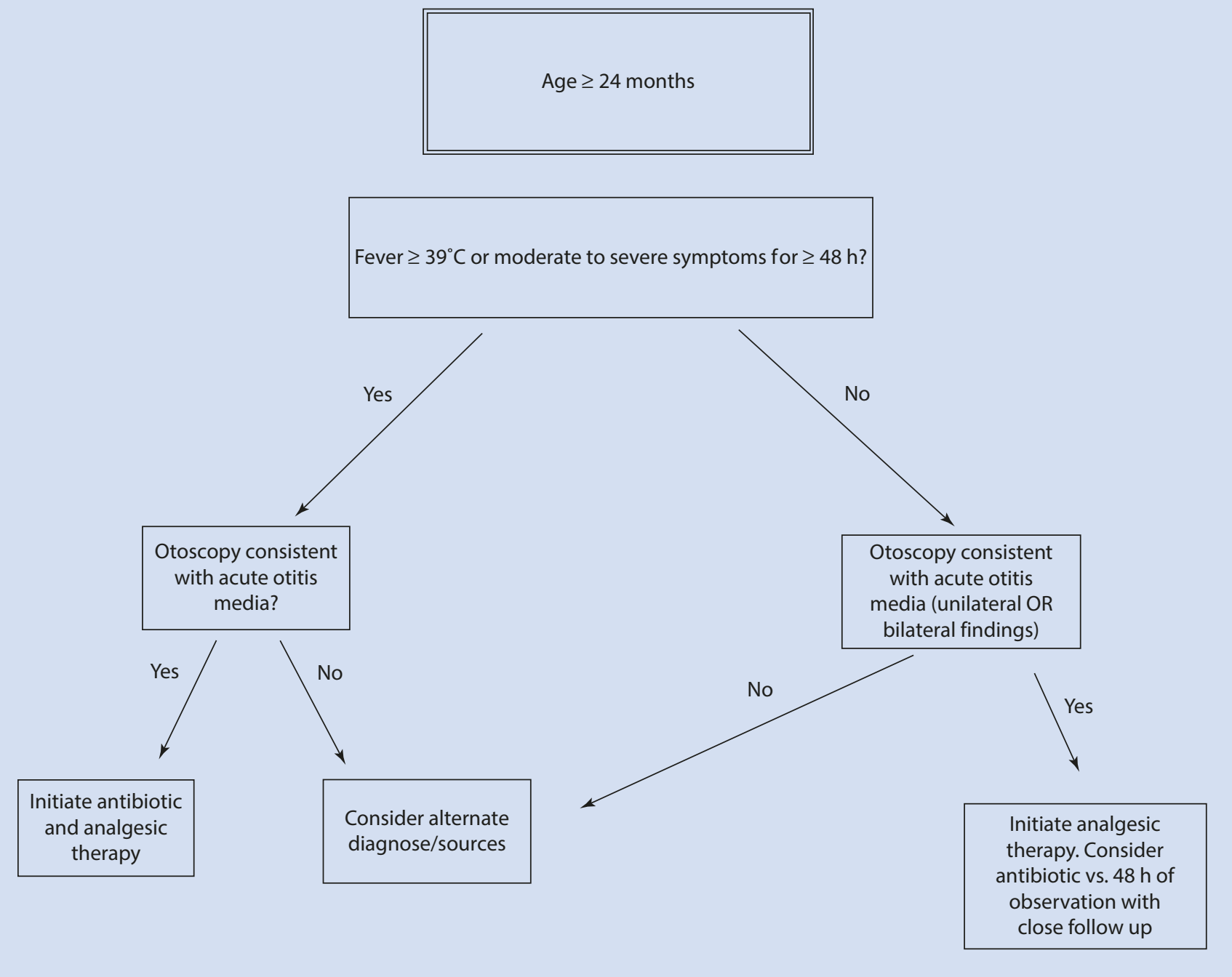

- Fig. 4.4 Treatment of AOM in patients $>24$ months of age

\section{Call Out Box 4.1}

Antibiotic Choices for the Treatment of Acute Otitis Media

- Uncomplicated AOM: high-dose amoxicillin

- The presence of a type 1 hypersensitivity to penicillin: azithromycin or clarithromycin

- The presence of a non-type 1 hypersensitivity to penicillin: 3rd-generation oral cephalosporin such as cefdinir.

- Previous antibiotic treatment in the past 4 weeks: amoxicillin plus clavulanic acid

- Amoxicillin failure at $48-72 \mathrm{~h}$ : amoxicillin plus clavulanic acid

- AOM with purulent conjunctivitis: amoxicillin plus clavulanic acid

- Amoxicillin plus clavulanic acid failure at 48-72 h: 3 rd-generation oral cephalosporin such as cefdinir or intramuscular ceftriaxone for 1, 2, or 3 days

- Ongoing emesis or adherence concerns: intramuscular ceftriaxone for 1,2 , or 3 days

\subsubsection{Complications of AOM}

Complications of AOM encompass spread of infection to structures adjacent to the middle ear and include mastoiditis, petrositis, venous sinus thrombosis, and central nervous system infection including brain abscess.

\subsubsection{Follow-Up}

Patients under treatment or observation for AOM should be reevaluated for the persistence or worsening of symptoms 48-72 $\mathrm{h}$ after the initial assessment. If symptoms are improving at $48-72 \mathrm{~h}$, short-term follow-up is not usually necessary. TM abnormalities seen on physical examination can persist for up to 12 weeks. 
Case Study

Practical Examples

Case 1

A 17-month-old girl presents with a

3-day history of rhinorrhea, cough,

fussiness, and sleeping less than

usual. Her highest recorded tempera-

ture over the course of illness is

37.7C. Her mother is concerned that

she may have an ear infection. On

physical examination she has clear rhinorrhea. Her left TM is unremarkable. The right TM is slightly opaque and has clear fluid behind it. The radial blood vessels of the TM are not injected. On pneumatic otoscopy, the right TM slightly reduced mobility. The heart and lung examinations are normal.

- What is the diagnosis?

- Are antibiotics warranted?

\section{Discussion}

This common clinical scenario offers a description of otitis media with effusion (OME). While the history of present illness includes upper respiratory infection symptoms and fussiness (a possible sign of ear pain in a toddler), and acute otitis media (AOM) is common in this age group, her physical examination findings are not consistent with AOM. The presence of a simple effusion in the middle ear space, in the absence of other signs of TM inflammation, is likely due to poor drainage of the eustachian tube in the setting of her current viral illness. She does not meet criteria for a diagnosis of AOM. Antibiotics are not warranted since the middle ear effusion is very unlikely to be due to a bacterial infection.

\section{Case 2}

A 4-year-old boy presents with a complaint that "my ear hurts!." His illness started with a cough and rhinorrhea 10 days earlier. He first developed fever 2 days ago. Despite this illness, the child has been able to participate in most of his usual activities. He has history of AOM with his last episode diagnosed 9 months ago. He has not taken antibiotics for any other reason since then. He has no medication allergies. On examination, the boy has clear rhinorrhea, mild pharyngeal injection, clear conjunctivae, and normal heart and lungs. His right external auditory canal is unremarkable. The right tympanic membrane shows intact bony landmarks and a small meniscus of clear effusion in the middle ear space. The left external auditory canal is unremarkable. His left TM is bulging and erythematous with a loss of the usual bony landmarks. The radial blood vessels are bright red. There is absence of mobility on pneumatic otoscopy.
What is the child's diagnosis?

- What is the correct treatment approach?

- If antibiotics are prescribed, what would be the first-line treatment option?

\section{Discussion}

The child's clinical history of otalgia with fever, preceded by an upper respiratory infection, along with physical examination findings of middle ear inflammation is consistent with left-sided acute otitis media. While the diagnosis is clear, the recommended approach to treatment offers two options. The boy is older than 24 months of age, his otalgia is not severe, and his fevers are under $39^{\circ} \mathrm{C}$. Under these circumstances, it is appropriate either to initiate antibiotic therapy or to wait and observe to determine whether his symptoms resolve on their own. Symptoms that persist or worsen during observation warrant the initiation of antibiotic therapy. The patient or the family may be given a "wait-and-see" prescription in this instance with instructions to fill it only if the symptoms do not improve in 48-72 $\mathrm{h}$. The boy in the vignette should be treated as a case of uncomplicated AOM without recent antibiotic exposure. Since he has no medication allergies, high-dose amoxicillin (80-90 mg/kg/ day) is the antibiotic treatment of choice.

\subsection{Sinusitis}

\subsubsection{Introduction}

Sinusitis refers to an infection of one or more of the paranasal sinus cavities. Acute upper respiratory viral infections that cause the common cold all involve the paranasal sinuses. The vast majority of these infections are self-limiting and do not require treatment with antibiotics. Acute bacterial rhinosinusitis is a more precise name for the condition caused by bacterial pathogens, and like AOM, it typically occurs when drainage is impaired secondary to the inflammation associated with a recent viral infection. Fortunately, only a small number of "colds" become complicated by acute bacterial sinusitis. While viruses and bacteria account for most infections of the paranasal sinuses, molds can cause some of the more severe disease. Immune-compromised patients are particularly vulnerable to these disfiguring, often fatal mold infections.

\subsection{Definitions}

\subsubsection{Sinusitis: Inflammation of the Paranasal Sinus Cavity Mucosa}

Acute bacterial rhinosinusitis (ABRS) - A bacterial infection leading to inflammation of the paranasal sinuses which resolves, with treatment, within 30 days

Subacute bacterial rhinosinusitis (SBRS) - A bacterial infection leading to inflammation of the paranasal sinuses which resolves in 30-90 days

Recurrent acute bacterial rhinosinusitis (RABR) - Bacterial infections leading to inflammation of the paranasal sinuses lasting fewer than 30 days but recurring 3 or more times in a 6-month period or 4 or more times during a 12-month period. Each infection responds well to treatment with antibiotics, and the patient experiences at least 10 symptom-free days in between episodes.

Chronic sinusitis - Inflammation of the paranasal sinuses for more than 90 days with persistent symptoms. This condition is most commonly associated with noninfectious processes such as environmental 
allergies, reactions to environmental pollutants, and gastroesophageal reflux disease. Patients with cystic fibrosis and ciliary dyskinesia are especially prone to chronic sinusitis secondary to noninfectious and infectious triggers.

\subsection{Basic Concepts}

\subsubsection{Anatomy and Pathophysiology}

The anatomy of the paranasal sinuses changes from birth, not becoming fully aerated until adulthood $[5,6]$. The ethmoid sinuses, present at birth, grow proportionally with the child. Maxillary sinuses, also present at birth, reach adult size by 4 years of age. The sphenoid sinuses begin to develop around 2 years of age, are functional by age 5 , and fully mature by age 12. Finally, the frontal sinuses are first evident by age 6-8 years, not fully maturing until early adulthood.

Acute bacterial rhinosinusitis is incited by predisposing factors such as viral upper respiratory infections, allergic rhinitis, environmental pollutants, nasal septum anomalies, other craniofacial anomalies, adenoidal hypertrophy, masses, polyps, or the presence of a foreign body. Risk factors include smoking, second-hand smoke exposure, and extensive dental disease. All of these conditions impede mucociliary clearance of sinus secretions. Since the sinus cavities are not sterile sites, stasis of secretions increases the likelihood that resident bacterial flora will overgrow resulting in acute bacterial rhinosinusitis $[5,6]$.

\subsubsection{Risk Factors}

Acute bacterial rhinosinusitis is most common between 4 and 7 years of age. Children in this age group are old enough to have relatively developed sinuses, yet young enough to experience frequent predisposing viral upper respiratory infections. These upper respiratory infections impede usual sinus clearance. Respiratory viral infections are even more common among children less than 2 years of age, but these younger children have less developed sinuses and wider ostia that facilitate drainage. In addition, younger children will often present earlier with AOM. Treatment of the AOM effectively treats any emerging sinus disease, therefore eliminating the opportunity for the development of acute bacterial sinusitis. A second peak of ABRS occurs during adulthood between 45 and 64 years of age. When the infections become recurrent, it is important to maintain a level of suspicion for the presence of contributing factors such as an anatomic blockage (polyps or a foreign body), gastroesophageal reflux disease, allergic disease, a humoral immune deficiency, cystic fibrosis, ciliary dysmotility, or any other condition that might lead to ongoing sinus inflammation and impaired sinus drainage $[5,6]$.

\subsubsection{Microbiologic Causes of Acute Bacterial Rhinosinusitis}

The optimal manner in which to determine the precise microbiology of acute bacterial rhinosinusitis is to obtain sinus fluid during the acute infection. Existing data, obtained decades ago, are based largely on studies where needle aspirates were performed on infected maxillary sinuses and the fluid sent to the microbiology laboratory for culture. The technique is modestly invasive and now seldom performed outside of academic settings, clinical trials, and severe or particularly enigmatic cases [7]. Not unexpectedly, the common causes of sinus infections are nearly identical to the list of pathogens that cause AOM since both processes arise from bacteria that normally inhabit the human upper respiratory tract at low concentrations.

The most common bacterial agents of ABRS include $S$. pneumoniae, non-typeable $H$. influenzae, and M. catarrhalis. S. pneumoniae and non-typeable $H$. influenzae are equally common in the post-pneumococcal vaccine era with each accounting for approximately $30 \%$ of cases. Infection with $M$. catarrhalis is less frequent at an estimated $10 \%$ of total cases $[5,6]$. Formal sinus aspirate studies have reported that approximately $30 \%$ of nasal sinus aspirates are culture negative for bacteria [7]. Less common causes of ABRS include $S$. aureus and $S$. pyogenes. Anaerobic bacteria have also been implicated either as a primary pathogen or as co-pathogens in several adult studies including Prevotella, Bacteroides, and Peptostreptococcus species. Anaerobic infection should be suspected if there is evidence of extension from dental disease into the sinuses.

\subsubsection{Approach to the Diagnosis}

The initial presentation of sinusitis varies. A careful history of the clinical course of the symptoms over time, the severity of symptoms, and the presence of fever are key details needed to help distinguish ABRS from the more common viral upper respiratory infections. Symptoms more suggestive of ABRS include fever, headache, facial pain and swelling, and halitosis, while cough, nasal congestion, and sore throat are typically seen during bacterial and viral infections [6] [ $\vee$ Call Out Box 4.2].

Call Out Box 4.2

A good general rule of thumb: If more than two mucous membranes are involved during a respiratory infection, the cause is viral, not bacterial. Bacterial infections of the upper respiratory tract tend to be localized. Viral infections can involve the eyes, ears, nose, sinuses, mouth, and pharynx simultaneously. 
- Table 4.1 IIIness characteristics that assist in distinguishing viral from bacterial sinusitis

\begin{tabular}{|c|c|c|}
\hline $\begin{array}{l}\text { Clinical } \\
\text { charac- } \\
\text { teristic }\end{array}$ & $\begin{array}{l}\text { Consider a viral } \\
\text { etiology }\end{array}$ & $\begin{array}{l}\text { Consider a bacterial } \\
\text { etiology }\end{array}$ \\
\hline $\begin{array}{l}\text { Progres- } \\
\text { sion of } \\
\text { symp- } \\
\text { toms over } \\
\text { time }\end{array}$ & $\begin{array}{l}\text { When severity peaks } \\
\text { on day } 3 \text { or } 4 \text { of the } \\
\text { illness, followed by } \\
\text { gradually improve- } \\
\text { ment with resolution } \\
\text { by day } 7-10\end{array}$ & $\begin{array}{l}\text { When symptoms } \\
\text { persist for more } 10 \text { days } \\
\text { When initial symptoms } \\
\text { are severe and the } \\
\text { patient has an ill } \\
\text { appearance for 3-4 } \\
\text { consecutive days } \\
\text { When severity peaks on } \\
\text { days 3-4, followed by } \\
\text { gradual improvement, } \\
\text { but on days 6-7, the } \\
\text { symptoms worsen } \\
\text { When new-onset fever, } \\
\text { headache, or facial pain } \\
\text { occurs on day } 7 \text { or later } \\
\text { of the illness }\end{array}$ \\
\hline Fever & $\begin{array}{l}\text { When fever is absent } \\
\text { or low grade only } \\
\text { When fever is present } \\
\text { only on days } 1 \text { and } 2 \text { of } \\
\text { the illness }\end{array}$ & $\begin{array}{l}\text { When any fever persists } \\
\text { for more than } 5 \text { days } \\
\text { When fever greater } \\
\text { than } 39^{\circ} \mathrm{C} \text { persists for } \\
3 \text { days or more } \\
\text { When there is new } \\
\text { onset of fever more } \\
\text { than } 7 \text { days into the } \\
\text { illness }\end{array}$ \\
\hline $\begin{array}{l}\text { Nasal } \\
\text { secretions }\end{array}$ & $\begin{array}{l}\text { When nasal discharge } \\
\text { is initially thin and } \\
\text { clear, becoming thick } \\
\text { and yellow-green } \\
\text { mid-illness then } \\
\text { resolves }\end{array}$ & $\begin{array}{l}\text { When nasal discharge } \\
\text { is initially thin and } \\
\text { clear, becoming thick } \\
\text { and yellow-green, and } \\
\text { persisting for more } \\
\text { than } 3 \text { days } \\
\text { When purulent } \\
\text { rhinorrhea is unilateral }\end{array}$ \\
\hline
\end{tabular}

The color of the nasal discharge is sometimes used to justify treatment with antibiotics. Viral upper respiratory tract infections usually begin with symptoms of clear, watery, nasal discharge. As the infection and associated inflammation proceed, the discharge becomes thicker and often takes on a yellow-green color. This change is expected during a viral infection and does not herald the presence of a bacterial process. The change in color and consistency is secondary to innate immune responses to the virus, with recruitment of inflammatory cells, including neutrophils. Neutrophil myeloperoxidase allows for some of the oxygenfree radicals to be converted to hypochlorite. It is the presence of this halogenated compound that renders the color change, not the presence of bacteria. Acute bacterial infections trigger the same innate immune inflammatory pathways, explaining why the discharge seen during a bacterial infection also has a yellow-green color. While the color and consistency of the nasal discharge is not helpful in distinguishing viral from bacterial sinusitis, several characteristics of the infection are. Table 4.1 lists some of the ways in
Call Out Box 4.3

Reliable Diagnostic Criteria for Acute Bacterial Rhinosinusitis History and physical examination must demonstrate the presence of sinus inflammation:

- Persistent Disease: Symptoms lasting 10-30 days without improvement (10-30 days is acute disease, $30-90$ days is subacute disease, 90 days or longer is chronic disease)

- OR

- Severe Disease: Severe symptoms at initial presentation with ill appearance, fevers higher than $39^{\circ} \mathrm{C}$, and persistent purulent rhinorrhea for 3-4 days - OR

- Worsening Disease: Symptoms showing improvement on days 6-7 of the illness followed by clinical worsening, including the presence of fever, cough, headache, and recurrence of nasal symptoms

which the viral and bacterial sinus infections can be differentiated from one another on clinical grounds.

Physical examination findings associated with ABRS include erythema and edema of the nasal turbinates, mucopurulent rhinorrhea, and the presence of postnasal drip. Facial pain and sinus tenderness to palpation are less reliable finding in children, but are quite useful indicators in adolescents and adults. None of the physical examination findings are completely specific to either a bacterial or viral infection. The clues obtained during the history of present illness are often the most important key.

Taking the history of present illness and physical examination findings into account, reliable diagnostic criteria for ABRS have been developed $[5,6]$ [ $\bullet$ Call Out Box 4.3].

Imaging is rarely useful in the evaluation of uncomplicated bacterial rhinosinusitis. Plain radiographs or computerized tomography imaging of the sinuses undertaken in the absence of compelling history and severe clinical course is likely to demonstrate misleading results. Mucosal thickening, sinus opacification, and air fluid levels may be present, but none of these findings are specific for bacterial underlying etiology. Sinus abnormalities are typically present on imaging in those who have simple viral upper respiratory infections [5]. In contrast, if bacterial rhinosinusitis is present AND complications or spread to adjacent structures is suspected, imaging is always indicated. Computerized tomography imaging of the sinuses and surrounding structures is first line to define the sinus anatomy and bony structures. If there is suspicion that the process has spread intracranially, magnetic resonance imaging of the brain should be considered.

Laboratory evaluation, as with imaging, is not warranted during the evaluation of uncomplicated ABRS. However, if the patient is ill appearing, is immunocompromised, and fails antibiotic treatment, or local extension of the infection is suspected, a sinus aspirate may be warranted. When obtained, the sample is typically sent for Gram stain, aerobic and anaerobic cultures with susceptibility testing. The presence of more than 10,000 colony forming units of a bacterium is considered clinically significant [5]. 


\subsubsection{Differential Diagnosis of Bacterial Rhinosinusitis}

The differential diagnosis of bacterial rhinosinusitis includes viral upper respiratory infection(s), allergic rhinitis, a local reaction to environmental pollutants, the presence of a nasal foreign body, and adenoidal hypertrophy or infection. If the chief complaint is a prolonged cough illness, infection with Bordetella pertussis should also be considered.

\subsubsection{Treatment of Bacterial Rhinosinusitis}

Treatment of bacterial rhinosinusitis is dependent on the clinical course of the illness. Subacute, recurrent, and chronic bacterial rhinosinusitis all warrant prompt antibiotic treatment. In contrast, "persistent" ABRS may be followed with clinical observation for up to 3 days. If symptoms continue or worsen during the observation period, antibiotic therapy is initiated. An observation course must be discussed in detail with patient and indications for returning to care must be explained $[5,6]$.

First-line antibiotic treatment options for ABRS include either amoxicillin or amoxicillin with clavulanic acid. The use of amoxicillin alone raises concerns regarding coverage of beta-lactamase-producing organisms such as non-typeable H. influenza, Moraxella catarrhalis, S. aureus, and most oral anaerobes. However, rates of clinical failure with amoxicillin alone are low, and amoxicillin boasts a long history of safety and tolerability. Amoxicillin-clavulanate provides broader antimicrobial coverage but can cause gastrointestinal side effects and is less palatable in suspension formulations used for young children. Therefore, either medication can be considered first line in the treatment of uncomplicated bacterial rhinosinusitis.

Certain conditions increase the risk for treatment failure on amoxicillin alone and warrant amoxicillin-clavulanate as the preferred first-line treatment. Included in this group are patients with immune-compromising conditions, those who are incompletely immunized, patients who have received antibiotics during the last 4 weeks or recently required hospitalization, and those with chronic bacterial rhinosinusitis [6]. If multidrug-resistant $S$. pneumoniae is suspected or confirmed as the etiologic pathogen, a third-generation cephalosporin (cefdinir, cefixime, or ceftriaxone) or quinolone class antibiotic, such as levofloxacin, may be necessary [6].

Patients with non-type 1 penicillin hypersensitivity will most likely tolerate advanced-generation cephalosporins without allergic manifestations. Those with type 1 penicillin allergy warrant treatment with a non-penicillin alternative such as levofloxacin, clindamycin, or linezolid [5].

If the illness severity warrants inpatient treatment, intravenous antibiotic regimens with antimicrobial spectra of activity that encompass all of the usual suspects include ampicillin-sulbactam, cefotaxime, ceftriaxone, or levofloxacin. In circumstances where intravenous treatment failure is suspected, multidrug-resistant $S$. pneumoniae should be considered as a potential cause while also referring the patient for surgical consultation to determine if a drainage procedure might facilitate improvement. While awaiting surgical advice, vancomycin can be added to the antibiotic regimen to broaden coverage that includes highly resistant $S$. pneumoniae and methicillin-resistant $S$. aureus. Intravenous metronidazole can also be considered to include coverage for the most resistant anaerobes including $B$. fragilis [5].

Adjunctive therapies to treat nasal symptoms, such as intranasal steroids, saline irrigation or lavage, mucolytics, decongestants, and antihistamines, lack a robust evidence basis, but remain common practices by patients and providers.

\subsubsection{Complications of Bacterial Sinusitis}

Complications of bacterial sinusitis occur when the infection extends to adjacent structures. Localized spread of sinus disease may result in periorbital or orbital cellulitis. Bony involvement may include the formation of a subperiosteal abscess. Orbital extension should be suspected when there is local tissue swelling and edema in the periorbital area. Intracranial extension can include septic cavernous sinus thrombosis, meningitis, brain abscess, or osteomyelitis of the frontal bone. Intracranial disease should be considered if the patient develops persistent and severe headache, mental status changes, focal neurologic examination findings, or persistent emesis.
Practical Example

Case 1

A 7-year-old boy presents to the office for a 14-day history of illness that began with cough, rhinorrhea, and complaints of a sore throat. Symptoms gradually worsened for the first few days, but began to improve on day 6 . His parents assumed this had been "a typical cold." On day 7 of illness, the boy's symptoms worsened.
His cough and rhinorrhea persisted, but secretions became thick and purulent again. He developed new facial pain, fevers to $39^{\circ} \mathrm{C}$, and general malaise. The day prior to the current office visit, the patient began to complain of right eye pain. On physical examination, he appears tired. The right eyelid and surrounding skin is red and swollen. His extraocular movements are intact, but he complains of discomfort when moving his right eye. He also complains of pain during gentle retropulsion. The conjunctivae are clear and there is no chemosis [ Call Out Box 4.4]. His nares show purulent rhinorrhea. The TMs are unremarkable. The oropharynx is erythematous with postnasal drainage present. The nasal turbinates are erythematous and edematous. 
- What characteristics of this presentation help you distinguish between viral and bacterial sinusitis?

- How would you classify his sinusitis?

- Would you prescribe antibiotics?

- Is any other evaluation warranted? Why or why not?

\section{Discussion}

The child's early illness included symptoms consistent with a "common cold" of viral etiology. Careful consider- ation of the clinical course shows that he developed new and more severe symptoms at the time one would expect a typical viral upper respiratory infection to resolve. The new symptoms of fever, headache, and purulent rhinorrhea suggest the evolution of acute bacterial rhinosinusitis (ABRS). In addition, his symptoms have persisted for 14 days. His presentation is best classified as "worsening" acute bacterial rhinosinusitis. This classification warrants initiation of antibiotic therapy. In addition, his report of new-onset eye pain and the findings of periorbital inflammation and discomfort with ocular movement raise suspicion for early extension of the infection to the orbital space. These findings should prompt consideration of inpatient antibiotic therapy and sinus and orbital computerized tomography imaging to evaluate for the presence of intraorbital infection.

\section{Call Out Box 4.4}

Chemosis is a term used to describe edema of the bulbar conjunctivae. It is usually easiest to appreciate at the limbus, where the bulbar conjunctiva is elevated above the plane of the cornea. Chemosis is a nonspecific finding, but does herald the presence of significant eye irritation.

\subsection{Mastoiditis}

\subsubsection{Introduction}

Mastoiditis is a suppurative infection of the mastoid air cells that most often presents as a secondary complication of otitis media. Its presentation and progression can be acute and require hospitalization and urgent surgical intervention. Routine vaccination against $S$. pneumoniae has reduced the incidence of AOM and led to a decrease in the frequency of mastoiditis in the past decade.

\subsection{Definitions}

Mastoiditis - A suppurative bacterial infection of the mastoid air cells Acute mastoiditis - Mastoiditis with symptom duration of less than 1 month

Coalescent mastoiditis - Infectious destruction of thin bony septae between mastoid air cells

Subacute or masked mastoiditis - A low-grade persistent middle ear and mastoid infection with destruction of the bony septae between the air cells

Chronic mastoiditis - Mastoiditis with symptoms exceeding 1 month in duration

\subsection{Basic Concepts}

\subsubsection{Pathophysiology}

The mastoid sinuses are networks of air cells divided by bony septae located in the posterior portion of both temporal bones. The space connects to the middle ear by a bony passage that is present at birth and increases in size until approximately 2 years of age. As the bony passages grow, they become lined with epithelium contiguous to the middle ear space. This continuity of epithelium allows for the possibility for infections of the middle ear to spread to the mastoid. The mastoids are bordered anteromedially by the middle ear and ossicles, the facial nerve, the bony portion of the EAC, the jugular vein, and the internal carotid artery and medially by the inner ear. The sigmoid sinuses are immediately posterior to the mastoids. The cranial fossa sits above, and the soft tissue and muscles of the lateral neck sit below the mastoids on both sides $[8,9]$.

Mastoiditis is invariably preceded by inflammation and effusion in the middle ear space. The presence of the effusion creates increased pressure between the middle ear and the mastoid air cells. Untreated spread of infection from the middle ear to the mastoid air cells results in resorption of the thin dividing bony septae and osteomyelitis of surrounding temporal bone.

\subsubsection{Risk Factors for the Development of Mastoiditis}

As mastoiditis is a secondary complication of AOM, the two infectious processes share the same set of risk factors. Those at risk for severe mastoiditis include patients with immunodeficiencies, functional or anatomic asplenia, and chronic heart or lung disease. Patients who are underimmunized or unimmunized against $S$. pneumoniae are also at increased risk. Individuals with cochlear implants are at risk for hardware-based infections [8].

\subsubsection{Microbiologic Causes of Mastoiditis}

The most common causes of acute mastoiditis include S. pneumoniae, S. pyogenes, and S. aureus (including methicillin-resistant strains). Less common etiologies include $P$. aeruginosa and other Gram-negative bacteria, non-typeable $H$. influenzae, and resident anaerobes of the oropharynx. Mycobacterium tuberculosis is an unusual cause 
of mastoiditis but should be considered as a possibility when the illness is diagnosed in individuals who live or have lived in TB endemic areas of the world.

Chronic mastoiditis is most frequently caused by P. aeruginosa. Other pathogens to consider when the infection presents as a chronic process include other Gram-negative bacilli, S. aureus, and anaerobes [8].

\subsubsection{Approach to the Diagnosis of Mastoiditis}

Acute mastoiditis presents with abrupt onset of fever, otalgia, and a red, swollen postauricular area. The pathophysiology dictates that these symptoms follow a recent middle ear infection. If the initial AOM was treated with antibiotics, there may be an interval of improvement followed by an abrupt worsening of symptoms. The physical examination reveals an abnormal middle ear consistent with AOM. Otorrhea is present in approximately half of the cases because the TM has perforated under the pressure of the infected space. The affected ear will show edema and erythema of the posterior auricular area. The pinna begins to protrude due to edematous displacement. As the infection progresses, fluctuance may develop in the postauricular area over the mastoid air cells.

Chronic mastoiditis is preceded by long-standing middle ear disease. Fevers and postauricular erythema and swelling become evident as the infection progresses. Chronic mastoiditis should be suspected when a patient experiences longstanding TM perforation with chronic otorrhea. Due to the chronicity of the process, the patient may also complain of hearing loss $[8,9]$.

The diagnosis of mastoiditis is based on the classic clinical signs and symptoms. Once the diagnosis is established, obtaining fluid for microbiologic culture is important to help guide definitive treatment. Samples can be obtained via tympanocentesis or, more commonly, from a mastoid sample collected during surgical debridement. Biologic samples should be sent for Gram stain and aerobic and anaerobic cultures with susceptibility testing. Acid-fast cultures should be requested in cases where tuberculosis is a possibility [8].

Imaging should be used in cases where mastoiditis has been diagnosed clinically as the findings will help to guide decisions regarding surgical care. In the absence of objective physical examination findings of mastoiditis, the presence of opacifications in the mastoid air cells is nonspecific since the finding is also common in uncomplicated serous otitis media and during uncomplicated AOM [10]. When mastoiditis is clinically suspected, computerized tomography scanning (CT) is preferred to evaluate the bony structures. Classic CT findings of mastoiditis include mastoid air cell opacifications, resorption of the bony septae, and coalescence of air cells. CT may also be indicated in patients with AOM that has been unresponsive to antibiotic therapy to rule out the development of secondary mastoiditis. If intracranial or soft tissue complications are suspected, magnetic resonance imaging (MRI) is preferred [8]. Symptoms suggestive of an intracranial complication include focal deficits on neurologic examination, hearing changes, vertigo, meningeal signs, or altered mental status.

\subsubsection{Differential Diagnosis of Mastoiditis}

Scalp infection, periauricular cellulitis, extension of otitis externa, and perichondritis of the auricle may all present with posterior auricular erythema and edema. Parotid swelling secondary to mumps may displace the pinna, but the direction of deviation is superiorly rather than inferiorly seen with mastoiditis [8].

The differential diagnosis of abnormal findings of the mastoid air cells by CT in the absence of objective physical examination findings of mastoiditis includes underaerated or sclerotic air cells due to prior AOM and OME.

\subsubsection{Treatment of Mastoiditis}

All cases of mastoiditis are potential candidates for surgical intervention, and all cases require treatment with antibiotics. Antibiotic alone may be appropriate if the illness presents with focal erythema and edema, but without fluctuance or signs of adjacent spread. Antibiotic therapy in combination with surgical intervention is necessary if fluctuance is present, there is a history of chronic otorrhea, or the patient has developed focal neurologic signs and symptoms such as vomiting, nystagmus, vertigo, or other signs of intracranial disease. Surgical intervention may be fairly straightforward with tympanocentesis or placement of tympanostomy tubes or quite extensive with open debridement of the infected mastoid tissue and adjacent structures.

The initial antibiotic therapy should be administered parenterally. The typical duration of therapy is a minimum of 3 weeks. Depending on the clinical response, the antimicrobial susceptibilities, and the likelihood of adherence, some patients may complete therapy with oral antibiotics. The firstline empiric choice antibiotic for the treatment of acute mastoiditis is typically a medication in the penicillin or cephalosporin class. If the initial presentation is severe, vancomycin is also utilized to ensure coverage against methicillinresistant $S$. aureus and highly resistant $S$. pneumoniae strains.

Chronic mastoiditis is treated empirically with a modified penicillin in combination with a $\beta$-lactamase inhibitor, such as ampicillin plus sulbactam or piperacillin plus tazobactam in combination with gentamicin. This combination provides coverage against, $P$. aeruginosa, and other Gram-negative bacilli and anaerobes. In patients with recurrent $\mathrm{AOM}$ and a strong suspicion of $P$. aeruginosa, piperacillin-tazobactam, ceftazidime, or cefepime can be used along with clindamycin [8].

The prognosis of mastoiditis is good if the disease is diagnosed and treated before a more serious complication develops. Increasing rates of morbidity and mortality are seen when the infection results in septic thrombosis of the cavernous sinus and/or spread to the temporal lobe of the brain [11]. 


\subsubsection{Complications of Mastoiditis}

As many as $20 \%$ of mastoiditis cases are associated with complications. Infections caused by $S$. pyogenes and S. aureus can be particularly aggressive. Interestingly, patients who have recently been treated with antibiotics for AOM have some of the highest rates of complications. This suggests that causative bacteria are particularly pathogenic or have become resistant to more conservative treatments [11]. Known complications involve contiguous spread of the infection to adjacent structures and include subperiosteal abscesses, Bezold's abscesses (infection of the sternocleidomastoid and trapezius muscle attachments), facial nerve paralysis, meningitis, subdural empyema, brain abscess, venous sinus thrombosis, labyrinthitis, temporal bone osteomyelitis, cerebrospinal fluid otorrhea, or conductive hearing loss secondary to destruction of the bony ossicles. Progressive disease can lead to systemic infection with bacteremia and distal septic emboli. A rare but classic complication known as Gradenigo's syndrome is diagnosed when there is petrositis with associated otitis media, ipsilateral medial rectus palsy, eye pain, and possible additional cranial nerve abnormalities [8].

\section{Case Study}

\author{
Practical Example \\ Case \\ A 2-year-old boy presents with \\ redness around his ear. Two weeks \\ prior, he had a "cold" with mild \\ symptoms of rhinorrhea and cough. \\ Seven days into his illness, he \\ developed fevers and began tugging \\ on his right ear. His provider diag- \\ nosed him with right $A O M$ and treated \\ him with a 10-day course of amoxicil- \\ lin. Two days into his course of \\ amoxicillin, he seemed to improve and \\ his fevers resolved. Two days after he \\ completed his antibiotic course, his \\ fevers returned and his mother \\ noticed redness and swelling behind \\ his right ear. On physical examination,
}

the boy appeared fussy and slightly ill. He had clear rhinorrhea. His left EAC and TM were unremarkable. His right TM showed dullness and erythema with visible pus behind it. His right EAC was mildly erythematous and edematous. Manipulation of the right pinna and palpation of the posterior auricular area were quite painful. The postauricular area was erythematous and edematous, but without fluctuance. The right pinna was slightly displaced anteriorly.

- What is the boy's diagnosis?

- Is imaging warranted?

- Should his management include antibiotics, surgery, or both?

\section{- Is inpatient or outpatient treatment more appropriate in this clinical setting?}

\section{Discussion}

The patient's age, preceding AOM, and physical examination findings are consistent with acute mastoiditis. $\mathrm{His}$ presentation was relatively recent in onset. He does not have fluctuance on physical examination or signs and symptoms of adjacent spread. A case could be made for imaging to confirm mastoid findings and rule out any progressive bony disease. His initial management should include treatment with intravenous antibiotics in the inpatient setting.

\subsection{Exercises}

Please refer to the supplementary information section for answers to these exercises.

Match the clinical scenario with the most likely pathogen. Each pathogen may be used once, more than once, or not at all.

\section{Pathogen}

1. Most common bacterial cause of acute otitis media

2. Classic bacterial cause of concurrent otitis and conjunctivitis

3. Most common vaccine preventable cause of otitis, sinusitis, and mastoiditis

4. Gram-positive cause of mastoiditis that produces a $\beta$-lactamase

5. An uncommon cause of mastoiditis associated with ossicle damage and hearing loss that should be considered in refugees from Africa and Asia
Characteristic finding
A. Mycobacterium tuberculosis
B. Streptococcus pyogenes
C. Moraxella catarrhalis

D. Haemophilus influenzae, nontypeable

E. Streptococcus pneumoniae

F. Staphylococcus aureus 


\subsection{Summary}

Otitis, sinusitis, and mastoiditis represent a spectrum of otolaryngologic infections that cause signs and symptoms overlapping with the ubiquitous viral upper respiratory infection or "common cold." Clinical guidelines for each stress careful consideration of diagnostic criteria, vigilance for the development of complications, the most appropriate antibiotic options, and when necessary, appropriate surgical interventions.

\section{References}

1. Rosenfeld R, Schwartz S, Cannon C, et al. Clinical practice guideline: acute otitis externa. Otolaryngol Head Neck Surg. 2006;150(1 Suppl):S1-S24. https://doi.org/10.1177/0194599813517083.

2. Gould J, Matz P. Otitis media. Pediatr Rev. 2010;31(3):102-16. https://doi.org/10.1542/pir.31-3-102.

3. Lieberthal A, Carroll A, Chonmaitree T, et al. Clinical practice guideline the diagnosis and management of acute otitis media. Pediatrics. 2013;131(3):e964-99. https://doi.org/10.1542/peds.2012-3488.

4. Moon M. Acute otitis media. In: Johns Hopkins Harriet Lane continuity clinic curriculum; 2014. https://ped.peaconline.org.
5. Wald E, Applegate K, Bordley C, et al. Clinical practice guideline for the diagnosis and management of acute bacterial sinusitis in children aged 1 to 18 years. Pediatrics. 2013;1071. https://doi. org/10.1542/peds.2013-1071.

6. Chow A, Benninger M, Brook I, et al. IDSA clinical practice guideline for acute bacterial rhinosinusitis in children and adults. Clin Infect Dis. 2012;54(8):e72-e112. https://doi.org/10.1093/cid/cis370.

7. Wald E, Milmoe G, Bowen A, et al. Acute maxillary sinusitis in children. N Engl J Med. 1981;304:749-54. https://doi.org/10.1056/ NEJM198103263014302.

8. Lewis K, Newman A, Cherry J. Mastoiditis. In: Feigin R, Cherry J, editors. Textbook of pediatric infectious diseases. 4th ed. Philadelphia: W.B. Saunders Company. p. 212-7.

9. Bunik M. Mastoiditis. Pediatr Rev. 2014;35(2):94-5. https://doi. org/10.1542/pir.35-2-94.

10. Sing S, Rettiganti MR, Quin C, et al. Incidental mastoid opacification in children on MRI. Pediatr Radiol. 2016;46(5):704-8. https://doi. org/10.1007/s00247-016-3545-7.

11. Carmel E, Curotta JH, Cheng AT. Prognostic effect of pre-and postadmission antibiotic treatment in paediatric acute mastoiditis. J Laryngol Otol. 2017;131(S1):S12-7. https://doi.org/10.1017/ S0022215116009063.

Further Reading

Schoem S, Darrow D, editors. Pediatric otolaryngology. AAP; 2012. 